\title{
Concurrence of the strengths and difficulties questionnaire and developmental behaviour checklist among children with an intellectual disability
}

\author{
L. J. Rice,' E. Emerson, ${ }^{2}$ K. M. Gray, ${ }^{4,5}$ P. Howlin, ${ }^{1,3}$ B. J. Tonge, ${ }^{4,5}$ G. L. Warner ${ }^{3}$ \\ \& S. L. Einfeld' \\ I Brain and Mind Centre, University of Sydney, Camperdown, New South Wales, Australia \\ 2 Centre for Disability Research and Policy, University of Sydney, Lidcombe, New South Wales, Australia \\ 3 Institute of Psychiatry, Psychology and Neuroscience, King's College, London, UK \\ 4 Centre for Developmental Psychiatry and Psychology, Department of Psychiatry, School of Clinical Sciences at Monash Health, \\ Monash University, Victoria, Australia \\ 5 Centre for Educational Development, Appraisal and Research, University of Warwick, Coventry, UK
}

\begin{abstract}
Background The Strengths and Difficulties Questionnaire (SDQ) is widely used to measure emotional and behavioural problems in typically developing young people, although there is some evidence that it may also be suitable for children with intellectual disability (ID). The Developmental Behaviour Checklist - Parent version (DBC-P) is a measure of emotional and behavioural problems that was specifically designed for children and adolescents with an ID. The DBC-P cut-off has high agreement with clinical diagnosis. The aim of this study was to estimate the relationship between DBC-P and SDQ scores in a sample of children with ID.

Method Parents of 83 young people with ID aged 4-17 years completed the parent versions of the
\end{abstract}

Correspondence: Professor Stewart Einfeld, Brain and Mind Centre, University of Sydney, 94 Mallet Street, Camperdown, New South Wales 2050, Australia. +612 9351 0885 (e-mail: stewart. einfeld@sydney.edu.au)
SDQ and the DBC-P. We evaluated the concurrent validity of the SDQ and DBC-P total scores, and the agreement between the DBC-P cut-off and the SDQ cut-offs for 'borderline' and 'abnormal' behaviour.

Results The SDQ total difficulties score correlated well with the DBC-P total behaviour problem score. Agreement between the SDQ borderline cut-off and the DBC-P cut-off for abnormality was high ( $83 \%$ ), but was lower for the SDQ abnormal cut-off $(75 \%)$. Positive agreement between the DBC-P and the SDQ borderline cut-off was also high, with the SDQ borderline cut-off identifying $86 \%$ of those who met the DBC-P criterion. Negative agreement was weaker, with the SDQ borderline cut-off identifying only $79 \%$ of the participants who did not meet the DBC-P cut-off.

Conclusion The SDQ borderline cut-off has some validity as a measure of overall levels of behavioural and emotional problems in young people with ID, and may be useful in epidemiological studies that include participants with and without ID. However, 
where it is important to focus on behavioural profiles in children with ID, a specialised ID instrument with established psychometric properties, such as the DBC-P, may provide more reliable and valid information.

Keywords concurrent validity, developmental behaviour checklist, intellectual disability, strength and difficulties questionnaire

The present investigation arose from the concurrence of two studies, the Longitudinal Study of Australian Children (LSAC), a population study (Gray \& Smart, 2009; Bayer et al., 20I I) and the Stepping Stones Triple P (SSTP) Project, a public health community intervention for parents of children with developmental disabilities aged 2-I2 years (Tellegen \& Sanders, 2013). To assess behavioural disturbance, the LSAC used the Strengths and Difficulties Questionnaire (SDQ), a measure designed for typically developing children; the SSTP project uses the Developmental Behaviour Checklist - Parent version (DBC-P), a measure specifically for children with disabilities. To assess the representativeness of children receiving SSTP, it was necessary to compare their level of behaviour problems with that of the Australian LSAC intellectual disability (ID) population. As a preliminary to this, our aim in the present study was to estimate the relationship between the SDQ and the DBC-P in children with ID.

The SDG (Goodman, I997) is a widely used measure of psychological well-being in children and adolescents aged $4-17$ years. It is cited in over 6000 publications and translated into almost a hundred languages. It has high concurrent validity with other measures of behavioural problems in typically developing children, including the Child Behaviour Checklist (Goodman \& Scott, I999; Muris et al., 2003) and the Rutter Parent and Teacher Questionnaire (Goodman, I997). Although designed for typically developing children, there is some evidence that the SDQ may also be suitable for young people with ID. In both typically developing and ID groups, inter-rater agreements between parent, teacher and child reports are similar (Beck et al., 2004; Emerson, 2005; Kaptein et al., 2008). The pattern of child self-reported SDQ scores is also consistent with ICD-Io psychiatric diagnoses in children with ID (Emerson, 2003, 2005).
The Developmental Behaviour Checklist primary carer version (DBC-P; Einfeld \& Tonge, 2002; Einfeld et al., 2006; Tonge \& Einfeld, 2003) was specifically developed for young people with ID aged 4-I 8 years, and is designed to measure a broad range of emotional and behavioural problems. The DBC-P has five factor analytically derived sub-scales producing a behavioural profile. The total scale score includes a clinical cut-off score, derived from a Receiver Operating Characteristics (ROC) analysis, to differentiate between individuals with and without clinically significant emotional and behavioural problems. It can also be used as a repeated measure to monitor intervention effectiveness. The DBC-P cutoff has high agreement with clinical diagnosis (Einfeld \& Tonge, 2002).

The aim of this study was to estimate the relationship between DBC-P and SDQ scores in the context of children with ID, (I) as a continuous measure and (2) as a binary classifier registering behaviour indicative of psychopathology. We examined the concurrent validity of scores on the DBC-P and SDQ as well as the positive, negative and overall agreement between DBC-P and SDQ cut-offs. High correlations between scores on the two measures as well as high agreement between the cutoffs would support the use of the SDQ in research with children with ID.

\section{Method}

\section{Participants}

Eighty-three parents of children with ID ( 55 males, 29 females; mean age Io.8 years, SD 3.0, range 4-I7) participated. Thirty-four participants $(40 \%)$ were recruited through two mental health service clinics in Australia (clinic group; 23 males, II females; mean age 9.4 years, SD 2.8, range 5-I5 years); the remainder $(50 ; 60 \%)$ were recruited from a large-scale survey of children with Down syndrome in England and Wales (Down syndrome group; 32 males, I8 females; mean age II.8 years, SD 2.8, range 8-I7 years). In total, 50 (60\%) participants had Down Syndrome; 28 (33\%) had a diagnosis of Autism Spectrum Disorder; $5(6 \%)$ had other disorders (Prader-Willi syndrome, Costello syndrome, Tetrasomy i8p, global developmental delay, cerebral palsy). 


\section{Measures}

The SDQ comprises 25 items, each rated on a 3-point scale ('not true', 'somewhat true' and 'certainly true'). There are five sub-scales: Conduct Problems, Emotional Problems, Hyperactivity/Inattention, Peer Relationships and Prosocial Behaviour. The first four sub-scales (20 items) are summed to generate the total difficulties score. Scores $\leq \mathrm{I} 3$ are classified as normal; scores I4-I6 are rated 'borderline'; scores $\geq \mathrm{I} 7$ indicate abnormality.

The DBC-P (Einfeld \& Tonge, 2002) has 96 items rated on a 3-point scale ('not true as far as you know', 'somewhat or sometimes true' and 'very true or often true'). There are five sub-scales (Disruptive Behaviour, Self-absorbed, Communication Disturbance, Anxiety and Social Relating Difficulties) and a total behaviour problem score provides an overall measure of behavioural/emotional disturbance; (Einfeld \& Tonge, 2002). The clinical cut-off for the DBC-P is 46 . This was determined using the Likelihood Ratio method by examining the quantitative power of the DBC-P to distinguish psychiatric 'cases' and 'non-cases' determined by clinical interviews conducted by psychiatrists with expertise in children with developmental disabilities. Sensitivity and specificity of the DBC-P clinical cutoff score were $83 \%$ and $85 \%$, respectively; area under the ROC curve was $92 \%$ (Einfeld \& Tonge, 2002). The DBC-P has high concurrent validity with the Maladaptive Behaviour Scale (Lambert \& Windmiller, I98I) and the Problem Behaviour section of the Scales of Independent Behaviours (Bruininks et al., I988). Internal consistency, criterion validity, test-retest reliability and inter-rater reliability are high (Einfeld \& Tonge, 2002).

In the Australian sample, the DBC and SDQ were completed at the same time in the clinic. In the UK sample, SDQs were completed by post; DBC's were completed some weeks later in interviews with parents.

\section{Statistical analysis}

Partial correlations were performed to explore the relationship between total scores on the DBC-P and SDQ, controlling for age, gender and syndrome (Down syndrome, Autism, Other). To test level of agreement between DBC-P and SDQ cut-offs (borderline and abnormal), we used overall, positive and negative agreement (Cicchetti \& Feinstein, I990). Overall agreement $=$ total proportion of agreements, positive and negative; positive agreement $=$ index of agreement for positive observations only; negative agreement $=$ index of agreement for negative observations only (Mackinnon, 2000). McNemar's test for $2 \times 2$ contingency tables was used to determine whether there was a statistical difference in the probabilities between the two tests. Each of these analyses was conducted separately for the two cohorts (clinic group and Down syndrome group) and then for the two groups combined.

\section{Results}

Table I summarises mean scores for each of the scales and sub-scales for all participants. As might be expected, total and sub-scale scores for the clinic sample were generally higher than in the Down syndrome group.

Correlations between total scores on the SDQ and DBC-P were high and significant (Table 2). Overall agreement between proportions scoring above cut-off on the two measures was high for the comparison between the DBC-P and SDQ borderline cut-off (0.83) but lower for the SDQ abnormal cut-off (0.75). Positive agreement between the measures was also higher for the DBC-P vs. SDQ borderline cut-off $(86 \%)$ than for the abnormal SDQ cut-off $(76 \%)$. Negative agreement was weaker, with $79 \%$ agreement for the SDQ borderline vs. DBC-P cut-off comparison and $73 \%$ for the SDQ abnormal vs. DBC-P comparison (Table 2). When the groups were analysed separately, in the clinic sample, the borderline SDQ cut-off had good positive agreement $(93 \%)$ but low negative agreement $(60 \%)$ with the DBC-P cut-off. In the Down syndrome group, overall agreement between the measures was lower, but negative agreement was slightly better than the positive agreement (Table I).

Proportions of participants scoring above and below the DBC-P and SDQ cut-offs are presented in Table 3. Overall, the SDQ abnormal cut-off classified fewer children as meeting criteria for emotional and behavioural problems than the DBC-P ( $46 \%$ vs. 60\%) although the difference was only significant for the Down syndrome group where only $26 \%$ scored above the SDQ abnormal cut-off $\left(X^{2}(I)=5.40, p=.002\right)$.

(C) 2017 MENCAP and International Association of the Scientific Study of Intellectual and Developmental Disabilities and John Wiley \& Sons Ltd 
L. J. Rice et al. - Concurrence of the SDQ and DBC

Table I DBC-P and SDQ total raw score and sub-scale raw scores

\begin{tabular}{lccc}
\hline & $\begin{array}{c}\text { Down syndrome group Mean (SD) } \\
(\mathbf{N}=\mathbf{5 0})\end{array}$ & $\begin{array}{c}\text { Clinic group Mean (SD) } \\
\mathbf{( N = 3 4 )}\end{array}$ & $\begin{array}{c}\text { Combined Mean (SD) } \\
\mathbf{( N = 8 4 )}\end{array}$ \\
\hline DBC-P total score & $46.9(26.8)$ & $69.8(3.3)$ & $56.1(27.7)$ \\
DBC-P sub-scales & $14.0(8.6)$ & $24.4(10.45)$ & $18.2(10.7)$ \\
Disruptive Behaviour & $15.3(11.9)$ & $19.4(9.0)$ & $17.0(10.9)$ \\
Self-absorbed & $6.9(4.8)$ & $9.5(4.5)$ & $7.9(4.8)$ \\
Communication & & & $5.7(3.7)$ \\
Disturbance & $4.2(2.9)$ & $7.9(4.0)$ & $5.0(3.9)$ \\
Anxiety & $3.8(3.7)$ & $6.7(3.3)$ & $17.13(6.8)$ \\
Social Relating Difficulties & $14.1(5.9)$ & $21.6(5.6)$ & $3.0(2.1)$ \\
SDQ total score & $2.4(1.8)$ & $3.9(2.2)$ & $3.3(2.7)$ \\
SDQ sub-scales & $2.0(1.6)$ & $5.2(2.8)$ & $6.6(2.5)$ \\
Conduct Problems & $5.7(2.5)$ & $8.0(1.8)$ & $4.1(2.5)$ \\
Emotional Problems & $4.0(2.6)$ & $4.2(2.3)$ & $5.2(2.8)$ \\
Hyperactivity/lnattention & $5.3(3.0)$ & $5.2(2.5)$ & \\
Peer Relationships & & & \\
Prosocial Behaviour & & & \\
\hline
\end{tabular}

DBC-P, Developmental Behaviour Checklist - Parent version; SDQ, Strengths and Difficulties Questionnaire.

Table 2 Agreement between SDQ and DBC-P cut-off scores

\begin{tabular}{lccc}
\hline \multicolumn{1}{c}{ Analysis } & Down syndrome & Clinic & Total \\
\hline $\begin{array}{l}\text { Partial correlation } \\
\text { Overall agreement }\end{array}$ & $0.66^{*}$ & $0.77^{*}$ & $0.74^{*}$ \\
$\begin{array}{l}\text { SDQ-Borderline } \\
\text { SDQ-Abnormal }\end{array}$ & 0.80 & 0.88 & 0.83 \\
Positive agreement & 0.70 & 0.82 & 0.75 \\
SDQ-Borderline & 0.77 & 0.93 & 0.86 \\
SDQ-Abnormal & 0.67 & 0.89 & 0.76 \\
$\begin{array}{l}\text { Negative agreement } \\
\text { SDQ-Borderline }\end{array}$ & 0.82 & 0.60 & 0.79 \\
SDQ-Abnormal & 0.77 & 0.57 & 0.73 \\
\hline
\end{tabular}

${ }^{\star} P<0.00$ I.

Note. Developmental Behaviour Checklist - Parent version (DBC-P) cut-off $=46$. Strengths and Difficulties Questionnaire (SDQ) abnormal cut-off $=17$; SDQ borderline cut-off $=14$.

When comparisons were based on the SDQ borderline cut-off, proportions scoring above the cutoffs were similar (DBC-P 60\%, SDQ 62\%).

\section{Discussion}

The SDQ (Goodman, 1997) is one of the most widely used measures of behavioural and emotional problems in young people. It is particularly useful in large scale population studies because of the relative ease and speed of completion. However, evidence of reliability and validity when used with cohorts of nontypically developing children is limited. The aim of the present study was to estimate the relationship between the SDQ and an alternative measure of emotional and behaviour problems, the DBC-P, which is specifically designed for individuals with ID and has been shown to have high agreement with clinical diagnosis. Data were obtained from two convenience samples: a group of children with Down syndrome involved in a large survey study and a group recruited through mental health clinics for children with developmental disabilities, most of whom also had a diagnosis of Autism Spectrum Disorder. As expected, the clinic sample scored higher than the Down syndrome group on almost all sub-scales. The SDQ abnormal cut-off classified fewer children as meeting criteria for emotional and behavioural problems than the DBC-P ( $46 \%$ vs. $60 \%$ ), but when comparisons were based on the SDQ borderline cutoff, proportions scoring above cut-off were similar (SDQ 62\%, DBC-P 60\%). The SDQ abnormal cutoff also had lower positive and negative agreement with the DBC-P than the SDQ borderline cut-off, suggesting that the borderline cut-off may be a better indicator of emotional and behavioural problems in children with intellectual disabilities.

(C) 2017 MENCAP and International Association of the Scientific Study of Intellectual and Developmental Disabilities and John Wiley \& Sons Ltd 
L. J. Rice et al. - Concurrence of the SDQ and DBC

Table 3 Proportions of participants scoring above and below the DBC-P and SDQ cut-offs

\begin{tabular}{|c|c|c|c|}
\hline & DBC-P & SDQ borderline & SDQ abnormal \\
\hline \multicolumn{4}{|c|}{ Down syndrome } \\
\hline Below cut-off & $28(56 \%)$ & $28(56 \%)$ & 37 (74\%) \\
\hline Above cut-off & 22 (44\%) & $22(44 \%)$ & $13(26 \%)$ \\
\hline McNemar's Test & & $X^{2}(1)=0.00, P=1$ & $X^{2}(I)=5.40, P=0.002$ \\
\hline \multicolumn{4}{|l|}{ Clinic sample } \\
\hline Below cut-off & $6(18 \%)$ & $4(12 \%)$ & $8(24 \%)$ \\
\hline Above cut-off & $28(82 \%)$ & $30(88 \%)$ & $26(76 \%)$ \\
\hline McNemar's Test & & $X^{2}(1)=1, P=0.32$ & $X^{2}(I)=0.67, P=0.4 I$ \\
\hline \multicolumn{4}{|l|}{ Combined } \\
\hline Below cut-off & 34 (40\%) & 32 (38\%) & 45 (54\%) \\
\hline Above cut-off & $50(60 \%)$ & $52(62 \%)$ & 39 (46\%) \\
\hline McNemar's Test & & $X^{2}(I)=0.29, P=0.59$ & $X^{2}(I)=5.76, P=0.02$ \\
\hline
\end{tabular}

The study has several limitations. Data are based on convenience samples involving mainly children with Down syndrome and autism, and although all were classified as having ID, details of intellectual functioning were not available. The samples were also recruited from very different sources, with the clinic group, as expected having higher levels of behaviour problems than the survey group. However, this provided a wide range of levels of emotional/behavioural problems, and the association between the DBC-P and the SDQ borderline score was confirmed in both cohorts. In the Down syndrome group, there was also a delay between parents' completing the SDQ and the DBC-P, which may have reduced the agreement between scores on the two measures. Finally, there was no direct assessment of the children's behavioural and mental state and the lack of comparison with clinical psychiatric diagnoses for the SDQ, and the concurrent direct psychiatric diagnosis and DBC-P data are derived from a previous study (Einfeld \& Tonge, I995).

Despite these caveats, our findings suggest that in epidemiological studies such as the LSAC population study (Gray \& Smart, 2009; Bayer et al., 20I I), which include both typically developing and atypical participants, the SDQ borderline cut-off has validity as a measure of overall levels of behavioural and emotional problems in children with ID. It is thus of potential value when comparisons are needed with the general population. However, the SDQ lacks ROC data for children with ID, which makes its sensitivity and specificity for detecting differences between individuals with and without clinically significant emotional and behavioural problems unknown. Other important psychometric properties for children with ID, for example, sensitivity to change and inter-rater agreement, are also missing. Thus, where it is important to focus on behavioural profiles in children with ID, as in the SSTP Project (Tellegen \& Sanders, 20I3), the DBC-P may be preferable. The DBC-P is also likely to be more informative in clinical settings when these features are particularly important

(Chandler et al., 2016).

\section{Conflict of Interest}

Professor Einfeld and Emeritus Professor Tonge are the authors of the Developmental Behaviour Checklist. They receive royalties for the German language version but derive no personal or financial benefit from the sale of any of other versions. No other authors reported any financial disclosures.

\section{References}

Bayer J. K., Ukoumunne O. C., Lucas N., Wake M., Scalzo K. \& Nicholson J. M. (20II) Risk factors for childhood mental health symptoms: national longitudinal study of Australian children. Pediatrics I28, eI-eI5.

Beck A., Daley D., Hastings R. P. \& Stevenson J. (2004) Mothers' expressed emotion towards children with and without intellectual disabilities. Fournal of Intellectual Disability Research 48, 628-38. 
L. J. Rice et al. - Concurrence of the SDQ and DBC

Bruininks R. H., Hill B. K. \& Morreau L. E. (1988) Prevalence and implications of maladaptive behaviors and dual diagnosis in residential and other service programs. Springer-Verlag, New York.

Chandler S., Howlin P., Simonoff E., O'sullivan T., Tseng E., Kennedy J. et al. (2016) Emotional and behavioural problems in young children with autism spectrum disorder. Developmental Medicine and Child Neurology 58, 202-8.

Cicchetti D. V. \& Feinstein A. R. (1990) High agreement but low kappa: I. The problems of two paradoxes. fournal of Clinical Epidemiology 43, 543-9.

Einfeld S. L., Piccinin A. M., Mackinnon A., Hofer S. M., Taffe J., Gray K. M. et al. (2006) Psychopathology in young people with intellectual disability. The fournal of the American Medical Association 296, I98I-9.

Einfeld S. L. \& Tonge B. J. (1995) The Developmental Behavior Checklist: The development and validation of an instrument to assess behavioral and emotional disturbance in children and adolescents with mental retardation. Fournal of Autism and Developmental Disorders 25, 8I-I04.

Einfeld S. L. \& Tonge B. J. (2002) Manual for the Developmental Behaviour Checklist: Primary carer version $(D B C-P)$ E teacher version (DBC-T). University of New South Wales, Sydney and Monash University, Melbourne.

Emerson E. (2003) Prevalence of psychiatric disorders in children and adolescents with and without intellectual disability. Fournal of Intellectual Disability Research 47, 5I-8.

Emerson E. (2005) Use of the Strengths and Difficulties Questionnaire to assess the mental health needs of children and adolescents with intellectual disabilities. Fournal of Intellectual and Developmental Disability 30, I4-23.

Goodman R. (1997) The Strengths and Difficulties Questionnaire: a research note. Fournal of Child Psychology and Psychiatry 38, 58I-6.
Goodman R. \& Scott S. (I999) Comparing the Strengths and Difficulties Questionnaire and the Child Behavior Checklist: is small beautiful? fournal of Abnormal Child Psychology 27, 17-24.

Gray M. \& Smart D. (2009) Growing Up in Australia: The Longitudinal Study of Australian Children: A valuable new data source for economists. Australian Economic Review 423, 367-76.

Kaptein S., Jansen D. E. M. C., Vogels A. G. C. \& Reijneveld S. A. (2008) Mental health problems in children with intellectual disability: use of the Strengths and Difficulties Questionnaire. Fournal of Intellectual Disability Research 52, I25-3I.

Lambert N. M. \& Windmiller M. (I98I) AAMD Adaptive Behavior Scale, School edn. McGraw Hill, Montley, CA.

Mackinnon A. (2000) A spreadsheet for the calculation of comprehensive statistics for the assessment of diagnostic tests and inter-rater agreement. Computers in Biology and Medicine 30, I27-34.

Muris P., Meesters C. \& van den Berg F. (2003) The Strengths and Difficulties Questionnaire (SDQ) -further evidence for its reliability and validity in a community sample of Dutch children and adolescents. European Child and Adolescent Psychiatry 12, I-8.

Tellegen C. L. \& Sanders M. R. (2013) Stepping Stones Triple P-Positive Parenting Program for children with disability: A systematic review and meta-analysis. Research in Developmental Disabilities 34, I556-7I.

Tonge B. J. \& Einfeld S. L. (2003) Psychopathology and intellectual disability: The Australian child to adult longitudinal study. International Review of Research in Mental Retardation 26, 6I-9I.

Accepted 6 September 2017

(C) 2017 MENCAP and International Association of the Scientific Study of Intellectual and Developmental Disabilities and John Wiley \& Sons Ltd 